\title{
TOXICIDAD DEL AIRE DE LA CIUDAD DE VILLA DEL ROSARIO EN MUESTRAS DE MATERIAL PARTICULADO-FRACCIÓN RESPIRABLE PM 2.5
}

\section{AIR TOXICITY OF THE CITY OF VILLA DEL ROSARIO IN SAMPLES OF PARTICULATE MATERIAL - RESPIRABLE FRACTION PM 2.5}

\author{
PhD. Alfonso Quijano Parra*, Esp. Mónica Juliana Quijano Vargas.* \\ MD. Lina Andrea Quijano Vargas**, MSc. Saury Tomas Manzano.*** \\ Universidad de Pamplona. \\ * Grupo de Investigación en Química. Laboratorio de Control de Calidad. \\ ** Facultad de Salud. Departamento de Medicina. Grupo de Investigación en Química. \\ *** Grupo de Investigación en Ingeniería Industrial. \\ Email: \{alfonsoquijanoparra, mojuquiva\}@unipamplona.edu.co; lianquiva@ hotmail.com
}

Resumen: En esta investigación se realizó el monitoreo de la fracción respirable PM 2.5 en una zona urbana de Villa del Rosario-Norte de Santander influenciada por el tráfico vehicular de fuentes móviles a gasolina y diésel. El objeto del presente trabajo es establecer la relación de la exposición al $\mathrm{PM}_{2.5}$, de los metales y los HAP encontrados en el aire de Villa del Rosario- Norte de Santander-Colombia y los posibles efectos sobre la salud humana.

Palabras clave: Cromatografía de gases, hidrocarburos aromáticos policíclicos, metales, $\mathrm{PM}_{2.5}$, toxicidad.

\begin{abstract}
In this research, the monitoring of the respirable fraction PM 2.5 was conducted in an urban area of Villa del Rosario-Norte de Santander influenced by vehicular traffic gasoline and diesel mobile sources. The purpose of this work is to establish the relationship between exposure to PM2.5, metals and PAHs found in the air of Villa del Rosario Norte de Santander-Colombia and the possible effects on human health.
\end{abstract}

Keywords: Gas chromatography, metals, polycyclic aromatic hydrocarbons, PM 2..5, toxicity.

\section{INTRODUCCIÓN}

En los últimos años, el material particulado ha sido ampliamente estudiado por su potencial impacto en la salud humana y la consiguiente necesidad de controlar y regular los contaminantes adheridos a la superficie de las partículas. Varios estudios indican que los efectos a la salud, dependen de las dimensiones de las partículas, de su concentración y sobre todo de la composición química. Las emisiones de los motores de las fuentes móviles a gasolina y diesel contribuyen al aumento de las emisiones de material particulado a la atmósfera
(Pey et al., 2009). El material particulado fracción respirable está dividido en dos grupos principales de acuerdo al tamaño de la partícula: $\mathbf{P M}_{10}$ y $\mathrm{PM}_{2.5}$ (Samara y Voutsa, 2005). Las partículas $\mathrm{PM}_{10}$ (diámetro aerodinámico $<10 \mu \mathrm{m}$ ) tienen la capacidad de penetrar y depositarse en la región traqueo bronquial del tracto respiratorio (Vinitketkumnuen et al., 2002). El $\mathrm{PM}_{2.5}$ es un indicador de las partículas finas (diámetro aerodinámico $<2.5 \mu \mathrm{m}$ ), se depositan en la región alveolar del tracto respiratorio, corresponde a una fracción agresiva debido a que éstas son respirables (Traversi et al., 2009), tienen mayor efecto en la 
salud, ya que pueden ingresar al sistema respiratorio depositándose en los alveolos pulmonares y llegar al torrente sanguíneo produciendo efectos negativos en la salud respiratoria en personas susceptibles (Dominici et al., 2006). En particular, las partículas finas $\left(\mathrm{PM}_{2.5}\right)$ causan un mayor impacto en la salud (Gamble, 1998; Oberdorster, 2001).

Algunos autores afirman que la exposición al PM 2.5 puede causar a corto y largo plazo efectos para la salud humana, como la muerte prematura, aumento de los síntomas respiratorios, reducción de la función pulmonar y alteraciones en el tejido pulmonar (IARC, 1984, 1987; Westerholm et al., 2001).

El material particulado atmosférico está compuesto por metales (Mahbub et al., 2011), compuestos orgánicos volátiles, hidrocarburos aromáticos Policíclicos (HAP), dibenzo-p-dioxinas y furanos, bifenilos policlorados, endotoxinas (Billet et al., 2008), en el área urbana, los metales pesados y los HAP están estrechamente asociados a las partículas finas, aumentando la toxicidad y la carcinogenicidad en el material particulado (Gutiérrez-Castillo et al., 2006; Risom et al., 2005).

Sin embargo, diversos componentes nocivos del material particulado, como los HAP, pueden contribuir o incluso potenciar los respectivos efectos adversos para la salud y han sido identificados como uno de los mayores tóxicos del aire en la atmosfera de las áreas rurales y urbanas. (Chen et al., 2006; Xinhui et al., 2008; Dimashki et al., 2007). Estos compuestos orgánicos contienen desde dos hasta ocho anillos aromáticos fusionados y provienen de la combustión incompleta de combustibles fósiles, madera y biomasa así como de la volatilización de productos del petróleo. (Orecchio y Papuzza, 2009).

La contaminación atmosférica es la vía primaria de la exposición humana a los HAP junto con la ingesta de alimentos contaminados. Es importante señalar que los HAP en general, son sospechosos de ser carcinogénicos en un grado u otro, aunque el nivel sea muy bajo. La principal característica que presentan sobre la salud, es su capacidad para inducir la formación de cáncer en los organismos expuestos. La actividad carcinógena se expresa a través de la biotransformación de estos compuestos a reactivos intermedios capaces de enlazarse covalentemente con el ADN e inducirlo a romper sus cadenas (Ravindra et al., 2006). El potencial mutágeno y carcinógeno de los HAP depende de los metabolitos que produzcan. (Koss y Tesseraux, 1999).

El objeto del presente trabajo es establecer la relación de la exposición al $\mathrm{PM}_{2.5}$, los metales y los HAP encontrados en el aire de Villa del RosarioNorte de Santander-Colombia y los posibles efectos sobre la salud humana.

\section{2.-MATERIALES Y MÉTODOS}

\subsection{Muestreo}

El $\mathrm{PM}_{2.5}$ se recogió en filtros pallflex de $47 \mathrm{~mm}$ de diámetro con un tamaño de poro de 2 micras. El muestreo fue realizado por un Partisol 2025 plus de la Ruprecht \& Patashnick que es un muestreador de bajo volumen, con un flujo volumétrico de $1 \mathrm{~m}^{3} / \mathrm{h}$ y un tamaño de entrada selectiva (SSI), permite la recogida de partículas con un diámetro aerodinámico menor de $2.5 \mu \mathrm{m}$. Los tiempos de muestreo del $\mathrm{PM}_{2.5}$, la temperatura ambiente, la presión ambiental y la humedad relativa en esta investigación son de 24 h.

\subsection{Sitio de Muestreo}

Se realizó el monitoreo de la fracción respirable $\mathrm{PM}_{2.5}$ en una zona urbana de Villa del Rosario localizada en las coordenadas $7^{\circ} 50^{\prime} 2^{\prime \prime} \mathrm{N}$ $72^{\circ} 28^{\prime} 27^{\prime \prime} \mathrm{O}$. Su altitud es de $440 \mathrm{msnm}$ y su zona horaria es UTC-5, este sector está influenciado por una vía nacional por donde circula el tráfico pesado de fuentes móviles a gasolina y diésel.

\subsection{Extracción de los HAP}

La extracción de la materia orgánica de los filtros de $\mathrm{PM}_{2.5}$ se realizó por ultrasonido en un baño ultrasónico (Branson 1510, modelo 1510R-MT) usando como solvente el Diclorometano. Los filtros provenientes del muestreo del $\mathrm{PM}_{2.5}$ se colocaron en un vaso de precipitado con $20 \mathrm{~mL}$ de diclorometano durante 15 minutos a temperatura ambiente; seguidamente se hicieron nueve repeticiones con el mismo volumen y tiempo.

Una vez obtenido el extracto, se concentró en un evaporador rotatorio de vacío, hasta alcanzar un volumen aproximado de $10 \mathrm{~mL}$, para obtener el extracto global. Posteriormente el extracto global se transfirió a viales, se sellaron y se mantuvieron en refrigeración y en la oscuridad hasta su análisis cromatográfico. 


\subsection{Identificación de Hidrocarburos Aromáticos Policíclicos (HAP)}

Para analizar los HAP presentes en el $\mathrm{PM}_{2.5}$ del aire de Villa del Rosario (extracto global), se utilizó un equipo de Cromatografía de Gases marca Agilent Technologies 6890A Plus Series II HewletPackard Plus con detector FID (Flame Ionization Detector). La columna utilizada es Restek Rxi-17 Sil MS, $30 \mathrm{~m}$ de longitud, $0.25 \mathrm{~mm}$ de diámetro, $0.25 \mu \mathrm{m}$ de diámetro interno (silarylene similar a $50 \%$ phenyl $/ 50 \%$ dimethyl polysiloxane).

Para la identificación de los HAP se utiliza el patrón de 18 hidrocarburos de Restek (catálogo \# 31841 EPA Method 8310 PAH Mixture). La identificación cualitativa de los HAP presentes en el extracto global se realizó de acuerdo a las siguientes condiciones: Temperatura del inyector $250{ }^{\circ} \mathrm{C}$, detector FID a $320^{\circ} \mathrm{C}$. Mezcla $(\mathrm{mL} / \mathrm{min})$ : Aire $400-\mathrm{H} 230-\mathrm{N} 2$ 45. Se inyectó $1 \mu 1$, modo splitless. Condiciones del horno: Temperatura inicial $65^{\circ} \mathrm{C}$ por $0.5 \mathrm{~min}$ y se incrementa de la siguiente manera: $15^{\circ} \mathrm{C} / \mathrm{min}$ hasta $200^{\circ} \mathrm{C}, 4^{\circ} \mathrm{C} / \mathrm{min}$ hasta $330^{\circ} \mathrm{C}$ durante $15^{\circ} \mathrm{C} / \mathrm{min}$. Tiempo de análisis por muestra $53.33 \mathrm{~min}$. Gas de arrastre Helio, flujo $20 \mathrm{~mL} / \mathrm{min}$.

\subsection{Extracción de los metales}

Se realiza siguiendo la metodología EPA IO-3.2, 1999.

\section{RESULTADOS Y DISCUSIÓN}

Se muestra el análisis estadístico realizado al monitoreo del material particulado recolectado en una zona urbana de Villa del Rosario. En la tabla 1 se muestra el análisis estadístico del PM $_{2.5}$ de Villa del Rosario. El análisis del PM 2.5 muestra que, en este caso la media es menor que la moda, y la mediana es menor que la media, se determina que es una distribución asimétrica negativa. Además se observa que la desviación típica es relativamente alta, esto demuestra un rango alto, teniendo un valor mínimo de 40 y máximo de 81 .

El análisis de la temperatura ambiente muestra una media de 27.985 , mediana 28 y moda 28 con desviación típica de 1.1452 con propiedad baja, reflejándose en el valor máximo (30) y mínimo (25.4), de esta manera se deduce que los datos tienden a una distribución normal (simétrica).
La presión ambiental muestra una media de 738.35 , mediana 738 y moda 738 con desviación típica de 1.203 con propiedad baja, reflejándose en el valor máximo (741) y mínimo (736), de esta manera se deduce que los datos tienden a una distribución normal (simétrica).

La Humedad relativa muestra una media de 738,35, mediana 738 y moda 738 con desviación típica de 1,203 con propiedad baja, reflejándose en el valor máximo (741) y mínimo (736), de esta manera se deduce que los datos tienden a una distribución normal (simétrica).

El interés en conocer los niveles ambientales de los HAP, radica fundamentalmente, en las propiedades carcinógenas, mutagénicas y teratogénicas que poseen algunos de estos compuestos, lo que los llevó a ser considerados como contaminantes prioritarios por la Agencia Norteamericana de Protección del Medioambiente (USEPA, 2004) y la IARC, 2008). Los contaminantes prioritarios han sido clasificados según el poder cancerígeno así: el Benzo (a) pireno como cancerígeno en humanos; el dibenzo (a,h) antraceno, dibenzo $(\mathrm{a}, \mathrm{l})$ pireno, clasificados como probables carcinógenos en humanos; el naftaleno, benzo (b,k,f) fluoranteno, criseno, benzo(a) antraceno, benzo(c) fenantreno, dibenzo[a,i] pireno, indeno[1,2,3 - c,d] pireno, 5 - metilcriseno fueron clasificados como posibles carcinógenos en humanos. (IARC, 2002); el fluoreno, fenantreno, y el pireno, se examinan como no clasificables. (Poster et al., 2006).

Las emisiones vehiculares han sido reconocidas como la fuente antropogénica más importante de HAP en el aire urbano. (Lim et al., 2007). Algunos autores han demostrado que los escapes de los motores de los vehículos son probablemente la fuente más importante de HAP actualmente detectada, varios estudios han demostrado que los HAP especialmente dañinos son aquellos con 5-6 anillos aromáticos se encuentran predominantemente en las partículas (PM), en su mayoría debido a su alto peso molecular y baja volatilidad (Fang et al., 2004; Culotta et al., 2005; Lu, Chen., 2008; Slezakova et al., 2011).

Para la identificación de los diferentes HAP presentes en el extracto global del PM 2.5 de Villa del Rosario extraído con diclorometano, se tomó como referencia la muestra patrón de 18 Hidrocarburos aromáticos policíclicos (EPA Method 8310 PAH Mix.), los compuestos presentes en la muestra patrón son: 1.- Naftaleno, 2.- 1-Metilnaftaleno, 3.- 2-Metilnaftaleno, 4.- 
Acenaftileno, 5.- Acenafteno, 6.- Fluoreno, 7.Fenantreno, 8.- Antraceno, 9.- Fluoranteno, 10.Pireno, 11.- Benzo(a)antraceno, 12.- Criseno, 13.Benzo(b) fluoranteno, 14.- Benzo(k) fluoranteno, 15.- Benzo(a) pireno 16.- Indeno(1,2,3-cd) pireno, 17.- Dibenzo(a,h) antraceno, 18.Benzo(ghi)perileno.

En la figura 1 se muestra el cromatograma de la materia orgánica del $\mathrm{PM}_{2.5}$, extraída con Diclorometano en Villa del Rosario.

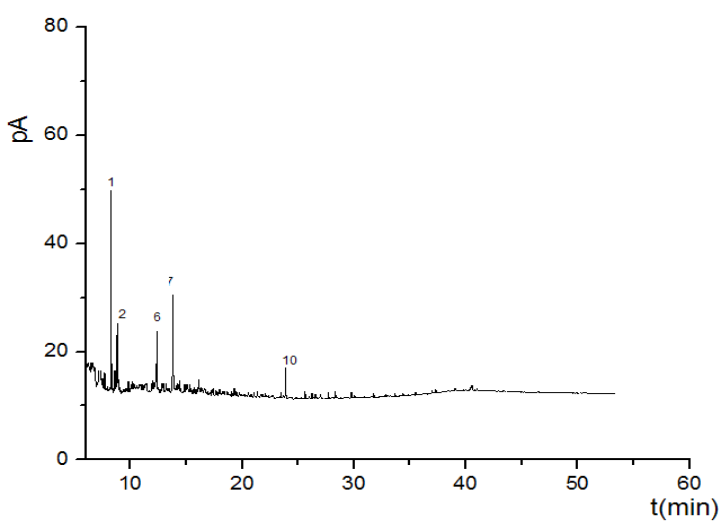

Fig. 1. Cromatograma de los HAP en muestras de $\mathrm{PM}_{2.5}$ del aire de Villa del Rosario

Como se muestra en este cromatograma, los HAP logrados identificar en el extracto global de la materia orgánica del aire de Villa del Rosario son: 1.-Naftaleno; 2.-1-Metil naftaleno; 6.-Fluoreno; 7.Fenantreno; 10.-Pireno.

En estudios realizados por el Grupo de Investigación en Química de la Universidad de Pamplona en el aire de Villa del Rosario se han encontrado además de los anteriores HAP, los siguientes: Benzo(a) antraceno; Dibenzo(a,h) antraceno; $\operatorname{Benzo}(\mathrm{g}, \mathrm{h}, \mathrm{i})$ perileno; Criseno; Benzo(b) fluoranteno; Benzo(k) fluoranteno y el Benzo(a) pireno.

Los HAPs producidos por la combustión de combustibles orgánicos son considerados como mutágenos (International Agency for Research on Cancer, 1989). El naftaleno es considerado como cancerígeno (IARC, 2002), el Dibenzo (a,h)antraceno es clasificado como probable carcinógeno en humanos; el Naftaleno, Benzo(a) antraceno; $\operatorname{Benzo}(\mathrm{g}, \mathrm{h}, \mathrm{i})$ perileno; Criseno; Benzo(b) fluoranteno; $\operatorname{Benzo}(\mathrm{k})$ fluoranteno, fueron clasificados como posibles carcinógenos en humanos y el Benzo(a) pireno como cancerígeno en humanos (IARC, 2002).El Programa Nacional de Toxicología Americano (NTP) anticipa que los contaminantes atmosféricos halllados en el aire de Villa del Rosario pueden ser considerados como carcinógenos en humanos.(RoC. NTP, 2009).

El fenantreno hallado en el aire de Villa del Rosario es característico de las emisiones del tráfico vehicular (Smith y Harrison, 1998), la mezcla de benzo(b,k) fluoranteno, hallada en el aire de Villa del Rosario está estrechamente relacionada con el diesel y los vehículos de motor de gasolina (Carreras et al., 2013), el benzo [g,h,i] perileno es un indicador de HAP emitidos por los escapes de los motores diesel y de gasolina. (Kuo et al., 1998).

Es necesario mencionar que los contaminantes prioritarios hallados en las muestras del aire (PM2.5) de Villa del Rosario, provienen exclusivamente de la combustión de las fuentes móviles que circulan con diesel y gasolina (Mi et al., 2001).

Los hidrocarburos policíclicos aromáticos nitrados (NHAP's) son derivados de los HAP, con dos o más anillos aromáticos fundidos formados por átomos de carbono, hidrógeno y nitrógeno. Los NPAH's se forman fundamentalmente como productos directos o indirectos de la combustión incompleta. El caso más representativo corresponde a los nitroisómeros más abundantes del pireno y del fluoranteno. Los gases de escape de motores diésel presentan altos niveles de 1 - nitropireno y 3 - nitrofluoranteno, siendo el 1 - nitropireno el más abundante (Tan et al., 2006), los nitro - NHAP son particularmente interesantes debido a su genotoxicidad.

Se ha demostrado que la principal causa de mutagenicidad del diésel y de las partículas del aire está asociada con estos. Según la Agencia Internacional de Investigación contra el Cáncer, algunos son posiblemente carcinógenos para los humanos (Hayakawa, 2009).

Los metales detectados en el aire de Villa del Rosario son: $\mathrm{Ca}, \mathrm{K}, \mathrm{Zn}, \mathrm{Cd}, \mathrm{Pb}, \mathrm{Fe}, \mathrm{Mn}$ y Cr. Los metales son conocidos por estar involucrados en la generación de especies reactivas de oxigeno (ROS) y radicales hidroxilo y bajo ciertas condiciones pueden causar daño oxidativo a las biomoléculas en los pulmones (Valavanidis et al., 2006). Los elementos característicos para fuentes móviles que utilizan el combustible diésel son el $\mathrm{Cr}$ y el Mn; metales como el Fe y el Mn son potencialmente genotoxicos (Lima et al., 2011). 


\section{CONCLUSIONES}

El tráfico automotor es probable que sea la principal fuente de contaminantes prioritario atmosféricos en la ciudad de Villa del Rosario. Es necesario mencionar que los HAP y los metales hallados en las muestras del aire $\left(\mathrm{PM}_{2.5}\right)$ de Villa del Rosario, provienen exclusivamente de la combustión de las fuentes móviles que circulan con diesel y gasolina, lo que nos habla a las claras de la toxicidad del aire de Villa del Rosario. Con estos estudios se pretende motivar a las autoridades Colombianas a que presten más atención a la calidad de los combustibles comercializados.

\section{REFERENCIAS}

Billet S., Abbas I., Le Goff J., Verdin A., Andre V., Lafargue P.E., Hachimi A., Cazier F., Sichel F., Shirali P., Garçon G. (2008). Genotoxic potential of polycyclic aromatic hydrocarbons-coated onto airborne particulate matter (PM ) in human lung epithelial A549 cells. Cancer Letters, 270(1), 144-155.

Carreras Hebe A., Calderón-Segura M.E., Gómez Arroyo S., Murillo-Tovar M.A., Amador Muñoz O. (2013). Composition and mutagenicity of PAHs associated with urban airborne particles in Córdoba, Argentina. Environmental Pollution. 2013; 178: 403-410.

Culotta L., Gianguzza A., Orecchio S. (2005). Leaves of nerium oleander L. as bioaccumulators of polycyclic aromatic hydrocarbons (PAH) in the air of Palermo (Italy). Extration GC-MS analysis, distribution, source, Polycyclic Aromat.Compd. 25:327-344.

Chen Y.C., Lee W.J., Uang S.N., Lee S.H., Tsai P.J. (2006).Characteristics of polycyclic aromatic hydrocarbon (PAH) emissions from a $\mathrm{UH}-1 \mathrm{H}$ helicopter engine and its impact on the ambient environment. Atmos. Environ. 40:7589-7597.

Dimashki M., Fadel A., Baba S., AlNouri M., Assad N. (2007). Measurements of polycyclic aromatic hydrocarbons (PAH), heavy metals, and gaseous pollutants in polluted airsheds over some Syrian cities. Proceedings of the Arab school of science \& technology workshop on ambient air quality, Kuwait.

Dominici F., Peng R.D., Bell M.L., Pham L., McDermott A., Zeger S.L., J.M. Samet J.M. (2006).Fine particulate air pollution and hospital admission for cardiovascular and respiratory diseases. J. Am. Med. Assoc. 295, 1127-1134.
Fang G.C., Chang C.N., Wu Y.S., Fu P.P.C., Yang I.L., Chen M.H. (2004) Characterization identification of ambient air and road dust polycyclic aromatic hydrocarbons in central Taiwan, Taichung. Sci. Total Environ. 2004; 327:135-146.

Gamble J.F. (1998). PM2.5 and Mortality in Longterm Prospective Cohort Studies: Cause-Effect or Statistical Associations?. Environ. Health Perspect. 106, 535-549

Gutiérrez-Castillo M.E., Roubicek D.A., CebriánGarcía M.E., De Vizcaya-Ruíz A., SordoCedeño M., Ostrosky-Wegman P.(2006). Effect of chemical composition on the induction of DNA damage by urban airborne particulate matter.Environ Mol Mutagen. Apr; 47(3):199-211.

Hayakawa K. (2009). Atmospheric Pollution and Its Countermeasure in East Asia from the Viewpoint of Polycyclic Aromatic Hydrocarbons. Journal of Health Science. 55: 870-878. )

IARC. (1984). Polynuclear Aromatic Compound. Part 1. Chemicals, Environmental and Experimental data. Monograph vol. 32.

IARC (1987). Overall Evaluation of Carcinogenicity: An Updating of IARC

Monographs, International Agency for Research on Cancer.

IARC.(1989). Monographs on the evaluation of carcinogenic risk to humans.Occupational Exposures in Petroleum Refining; Crude Oil and Major Petroleum Fuels, Vol. 45. Lyon: IarcPress, 1989.

IARC. (2002). Some traditional herbal medicines, some mycotoxins, naphthalene and styrene, IARC Monogr. Eval. Carcinog. Risk. Hum. 2002; 82:367.

IARC. (2008) Monographs on the evaluation of carcinogenic risk to human: Air pollution, Part 1, vol. 92, Some Non-Heterocyclic PAHs and related industrial exposures.2008.

Koss G., Tesseraux I. Toxicology. San Diego: Academic Press; 1999: 603-44.

Kuo C.Y., Cheng TW, Chen C.Y., Lee H.(1998). Correlation between theamounts of polycyclic aromatic hydrocarbons and mutagenicity of airborne particulate samples from Taichung City. Taiwan. Environ. Res. 78:43-49

Lima P.D., Vacconcellos M.C., Montenegro R.C., Bahia M.O., Costa E.T., Antunes L.M., Burbano R.R. (2011). Genotoxic effects of aluminun, iron and manganese in human cells and experimental systems: a review of the Literatura. Hum. Exp. Toxicol. 30(10): 14351444. 
Lim M.C.H., Ayoko G.A., Morawska L., Ristovski Z.D., Jayaratne E.R. (2007). Influence of fuel composition on polycyclic aromatic hydrocarbon emissions from a fleet of inservice passenger cars. Atmos. Environ, 41:50-160.

Lu H., Chen S. (2008). Pollution level, phase distribution and health risk of polycyclic aromatic hydrocarbons in indoor air at public places of Hangzhou, China. Environ. Pollut. 152: 569-575.

Mahbub P., Goonetilleke A., Egodawatta P.K., Yigitcanlar T., Ayoko G.A. (2011). Analysis of build-up of heavy metals and volatile organics on urban roads in Gold Coast,Australia. Water Science and Technology ,63 (9), 2077-2085)

Mi H.H., Lee W.J., Tsai P.J., Chen C.B. (2001). A comparison on the emission of polycyclic aromatic hydrocarbons and their corresponding carcinogenic potencies from a vehicle engine using leaded and lead-free gasoline. Environ. Heal. Perspect. 109 (12):1285-1290.

Oberdorster G. (2001). Pulmonary effects of inhaled ultrafine particles Int. Arch. Occup. Environ. Health 74, 1-8.

Orecchio S., Papuzza V. (2009). Levels, fingerprint and daily intake of polycyclic aromatic hydrocarbons (PAHs) in bread baked using wood as fuel, J. Hazard. Mater. 164:876-883.

Pey J., Querol X., Alastuey A., Rodriguez S., Putaud J. P., Van Dingenen R. (2009). Source apportionment of urban fine and ultra fine particle number concentration in a Western Mediterranean city. Atmospheric Environment, 43, 4407-4415.

Poster D. L., Schantz M. M., Sander L. C., Wise S. A. (2006). Analysis of polycyclic aromatic hydrocarbons (PAHs) in environmental samples: a critical review of gas chromatographic (GC) methods. Anal. Bioanal. Chem.386:859-881.

Ravindra K., Bencs L., Wauters E., de Hoog J., Deutsch F., Roekens E., Bleux N. et al.(2006). Seasonal and site-specific variation in vapour and aerosol phase PAHs over Flanders (Belgium) and their relation with anthropogenic activities.Atmospheric Environment.40 (4):771785).

Risom L., P. Møller, S. Loft.(2005). Oxidative stress induced DNA damage by particulate air pollution.Mutat. Res. Fundam. Mol. Mech. Mutagen, 592, 119-137.

RoC. National toxicology program (NTP): Report on carcinogens. U. S. Department of health and human services, public health service. National toxicology program (NTP). 2009.
Samara C \& Voutsa, D. (2005) Size distribution of airborne particulate matter and ssociated heavy metals in the roadside environment.Journal Chemosphere, 59, 1197-1206.

Slezakova K, Castro D, Delerue-Matos C., Alvim-

Ferraz M.C., Morais S., Pereira M.C. (2011). Air pollution fromtraffic emissions in Oporto, Portugal: Health and environmental implications. Microchem. J. 99:51-59

Smith D.J.T.,Harrison RM. (1998). Polycyclic Aromatic Hydrocarbons in Atmospheric Particles, in: R.M. Harrison, R. Van Grieken (Eds.), Atmospheric Particles,John Wiley \& Sons, New York, 1998.

Tan JH, Bi XH, Duan JC, Rahn KA, Sheng GY, Fu JM.(2006). Seasonal variation of particulate polycyclic aromatic hydrocarbons associated with PM10 in Guangzhou, China. Atmos. Res. 80(4):250-262.

Traversi D., Degan R., De Marco R., Gilli G., Pignata C., Villani S., Bono R. (2009). Mutagenic properties of PM urban pollution in Northern Italy: the nitro- compounds contribution. Environment International ,35, 905-910.)

Tsai P.J., Shih T.S., Chen H.L., Lee W.J., Lai C.H., Liou S.H. (2004). Assessing and predicting the exposures of polycyclic aromatic hydrocarbons (PAHs) and their carcinogenic potencies from vehicle engine exhausts to highway toll station workers.Atmos. Environ. 38:333-343.

U.S. EPA. Air quality criteria for particulate matter. Washington, 2004.

Valavanidis A., Kostantinos F., Thomais V., Bakeas E.B., Triantafillaki S., Paraskevopoulou V., Dassenakis M. (2006). Characterization of atmospheric particulates, particle bound transition metals and polyciclic aromatic hydrocarbons of urban air in the centre of Athens (Greece).Chemosphere 65:760-768

Vinitketkumnuen U, Kalayanamitra K,Chewonarin T, Kamens R. (2002). Particulate matter, $\mathrm{PM}_{10}$ \& PM levels, and airborne mutagenicity in Chiang Mai, Thailand. Mutation Research $e$ Genetic Toxicology and Environmental Mutagenesis 519, 121-131

Westerholm R, A. Christensen, M. T"ornqvist, L. Ehrenberg, U. Rannug,M. Sj"ogren, J.Rafter, C.. (2001). Comparison of exhaust emissions from Swedish environmental classified diesel fuel (MK1) and European Program on Emissions, Fuels and Engine Technologies (EPEFE) reference fuel: a chemical and biological characterization, with viewpoints on cancer risk. 
Soontjens,J. Alm’en, K. Gr“agg. Environ. Sci \& Technol. 35 (2001) 1748-1754

Xinhui Bi, Bernd RTS, Sheng Guoying, Ma Shexia, Jiamo Fu. (2008). Composition and major sources of organic compounds in urban aerosols. Atmos. Res. 88:256-265.

\section{ANEXOS}

\section{Material Particulado.}

\section{Tabla 1: Material Particulado}

\begin{tabular}{|ll|r|}
\multicolumn{2}{l}{ Material_particulado } & \multicolumn{2}{l}{ Estadísticos } \\
\hline N & Válidos & 34 \\
Media & Perdidos & 0 \\
Error tip. de la media & & 57,103 \\
Mediana & & 1,5406 \\
Moda7 & & 57,000 \\
Desv. típ. & & 58,0 \\
Varianza & & 8,9830 \\
Asimetría & 80,694 \\
Error típ. de asimetria & & 760 \\
Curtosis &, 403 \\
Error típ. de curtosis & & 1,273 \\
Rango & 0,788 \\
Minimo & & 41,0 \\
Máximo & 40,0 \\
Suma & & 81,0 \\
& & 1941,5 \\
Percentiles & 25 & 49,000 \\
& 50 & 57,000 \\
\hline
\end{tabular}

En la figura 1 se muestra el análisis estadístico realizado al $\mathrm{PM}_{2.5}$ de Villa del Rosario.

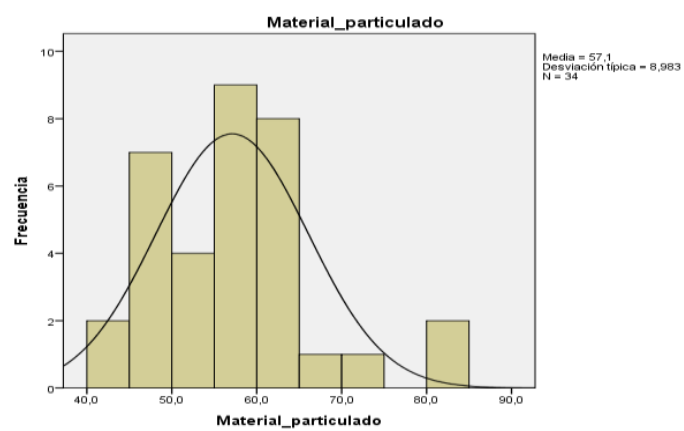

Fig. 1. Material particulado.

\section{Temperatura ambiente.}

Tabla 1: Temperatura ambiente

\begin{tabular}{|c|c|c|}
\hline & Estadisti & \\
\hline Temperatura_ambiente & & \\
\hline & Válidos & \\
\hline & Perdidos & \\
\hline Media & & 27,985 \\
\hline $\begin{array}{l}\text { Error típ. de la media } \\
\text { Mediana }\end{array}$ & & $\begin{array}{l}0,1964 \\
28,000\end{array}$ \\
\hline Moda & & 28,0 \\
\hline Desv. tip. & & 1,1452 \\
\hline Varianza & & 1,312 \\
\hline Asimetría & &,- 341 \\
\hline Error típ. de asimetría & & ,403 \\
\hline Curtosis & &,- 405 \\
\hline Error típ. de curtosis & &, 788 \\
\hline Rango & & 4,6 \\
\hline Minimo & & 25,4 \\
\hline Máximo & & 30,0 \\
\hline Suma & & 951,5 \\
\hline & 25 & 27,275 \\
\hline Percentiles & 50 & 28,000 \\
\hline & 75 & 29,000 \\
\hline
\end{tabular}

En la figura 2 se muestra el análisis realizado al monitoreo de la Temperatura ambiente de Villa del Rosario.

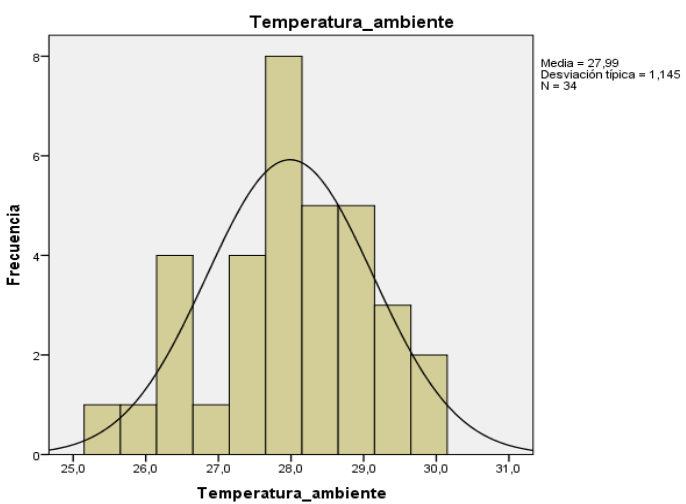

Fig. 2. Temperatura ambiente.

\section{Presión}

\begin{tabular}{|c|c|c|}
\hline \multicolumn{3}{|c|}{ Tabla 3: Presión } \\
\hline \multicolumn{3}{|l|}{ Presión } \\
\hline & Válidos & 34 \\
\hline & Perdidos & 0 \\
\hline Media & & 738,35 \\
\hline Error típ. de & &, 206 \\
\hline Mediana & & 738,00 \\
\hline Moda & & 738 \\
\hline Desv. típ. & & 1,203 \\
\hline Varianza & & 1,447 \\
\hline Asimetría & &, 369 \\
\hline Error típ. de & &, 403 \\
\hline Curtosis & &,- 593 \\
\hline Error típ. de & &, 788 \\
\hline Rango & & 5 \\
\hline Mínimo & & 736 \\
\hline Máximo & & 741 \\
\hline Suma & & 25104 \\
\hline & 25 & 737,75 \\
\hline Percentiles & 50 & 738,00 \\
\hline & 75 & 739,25 \\
\hline
\end{tabular}

En la figura 3 se muestra el análisis realizado a la presión ambiental durante el monitoreo de PM 2.5 de Villa del Rosario.

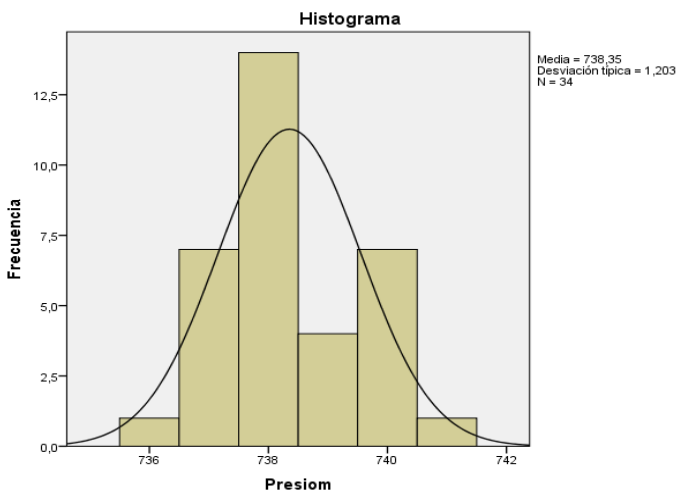

Fig. 3. Presión. 